Chronic Obstructive Pulmonary Diseases: Journal of the COPD Foundation

\author{
Journal Club
}

\title{
Journal Club - Bronchiectasis/COPD Overlap: Syndrome Versus Treatable Trait?
}

\author{
Ron Balkissoon, MD, MSc, DIH, FRCPC ${ }^{1}$
}

Abbreviations: chronic obstructive pulmonary disease, COPD; phosphodiesterase 4, PDE4; nontuberculous mycobacterial, NTM; computed tomography, CT; pulmonary artery to aorta, PA/A; right heart catheterization, RHC; inhaled corticosteroids, ICSs; long-acting beta2-agonists, LABA

Citation: Balkissoon R. Journal club-bronchiectasis/COPD overlap: syndrome versus treatable trait? Chronic Obstr Pulm Dis. 2019;6(2):193-199. doi: https://doi.org/10.15326/jcopdf.6.2.2019.0134

\section{Denver, Colorado}

\section{Address correspondence to:}

Ron Balkissoon, MD, MSc, DIH, FRCPC

balkissoonr@njhealth.org

\section{Keywords}

bronchiectasis; chronic obstructive pulmonary disease; COPD; overlap syndrome

\section{Introduction}

Chronic obstructive pulmonary disease (COPD) patients that have evidence of bronchiectasis demonstrate greater symptom severity, more frequent bronchial infections and exacerbations, lower lung function and increased mortality. ${ }^{1}$ While the association has been recognized, it is not clear if there is any causal relationship between having COPD and developing bronchiectasis. Certainly, the fact that COPD is associated with a high risk for lower respiratory infection ${ }^{2}$ and/or dysbiosis ${ }^{3}$ may be part of the explanation but not all patients with COPD develop bronchiectasis. Bronchiectasis has been defined as a dilation of the airways that persists beyond the acute responses to various insults such as infections, aspiration and toxic inhalations. The most accepted criteria as defined by Naidich include direct signs of a bronchial-arterial ratio $>1$, lack of bronchial tapering, visualization of peripheral bronchi within $1 \mathrm{~cm}$ of the costal pleura or in contact with the mediastinal pleura and indirect signs such as peribronchial thickening, mucus plugging, mosaic pattern, central lobular nodules, tree in bud nodules, focal areas of air trapping and atelectasis/consolidation). ${ }^{4}$ Bronchiectasis is known to develop and progressively worsen over time secondary to a number of conditions including cystic fibrosis, alpha-1 antitrypsin deficiency, primary ciliary dyskinesia, allergic bronchopulmonary mycosis, immunoglobulin deficiencies and post obstructive pneumonia secondary to tumors, foreign bodies and lymphadenopathy. Other less common causes include rheumatoid arthritis and Sjogren's disease, connective tissue disorders such as tracheobronchomegaly (Mounier Kuhn), Marfan's disease, cartilage deficiency (Williams Campbell syndrome), inflammatory bowel disease, HIV, Job's syndrome, and malignancies such as, chronic lymphocytic lymphoma and graft-versus-host disease, as well as yellow nail syndrome and Young's syndrome. ${ }^{5}$ It has been proposed that bronchiectasis develops as a result of these various disorders leading to structural and functional changes in the airways that increase susceptibility to chronic bronchial infections secondary to potentially pathogenic microorganisms. The persistent presence of these organisms causes chronic inflammation, airway remodeling and further damage to local defense mechanisms further enabling these potentially pathogenic microorganisms to persist in the airways despite the repeated administration of antibiotics thus creating a vicious cycle as proposed by Cole et al. ${ }^{6}$ With this backdrop it is not surprising that there has been recognition of a high prevalence of bronchiectasis in the COPD population with rates ranging from $4 \%$ to $72 \% .{ }^{7}$ The broad prevalence range reported is likely a result of several factors including differences in patient characteristics, evaluations 
during times of exacerbation versus clinical stability, and whether the prevalence of bronchiectasis was part of a primary or secondary analysis of data. Suffice it to say that the true prevalence of bronchiectasis/ COPD overlap remains unknown. Regardless, similar associations have also been noted in asthma ${ }^{8,9}$ and it has also been recognized that up to $75 \%$ of bronchiectasis patients demonstrate signs of upper airway disease and report severe daily symptoms of nasal congestion, facial pain and/or loss of smell. ${ }^{10}$

Considering the various combinations and permutations of these overlaps, several COPD phenotypes could be generated but it is debatable as to whether defining such overlap syndromes will lead to particularly unique treatment paradigms. Rather, it may be best to simply look at the various components as treatable traits and optimize treatment for each component as outlined by Polverino and colleagues in an excellent review recently published. ${ }^{1}$ Polverino and colleagues also point to many questions that need to be addressed regarding the COPD/bronchiectasis overlap. We need a set of updated consensus criteria for defining radiological and clinical bronchiectasis in patients with COPD to better establish prevalence and the potential prognostic value of identifying bronchiectasis in COPD patients. New methods to diagnose bronchiectasis independent of vessel diameter are needed, as the classic definition, airway diameter/blood vessel diameter ratio greater than 1 , can be misleading as cardiovascular comorbidities can cause increases in blood vessel diameter. There are also no specific biomarkers linking COPD to bronchiectasis such as possible genetic and epigenetic phenomena that may influence susceptibility to infections and response to treatment that renders only a subgroup of COPD patients to develop bronchiectasis. Other questions remain with regard to alterations of the microbiome, so called dysbiosis, ${ }^{3}$ that may be related to microaspiration ${ }^{11}$ and/or the potential impacts of inhaled corticosteroid use and frequent antibiotic use. ${ }^{12-15}$ Inhaled corticosteroids are often recommended for COPD patients with frequent exacerbations whereas recent guidelines in the Cochrane review have concluded that there is insufficient evidence to recommend the use of inhaled steroids in adults with stable bronchiectasis except in specific conditions where the possible benefits in exacerbation reduction outweigh the risks. ${ }^{16}$ Other areas for study include interventions that may help prevent progression of disease and exacerbations, including the role of other anti-inflammatory molecules such as phosphodiesterase 4 (PDE4) inhibitors or prophylactic antibiotic treatment either systemically or by inhalation. ${ }^{1}$ These are important questions to address in the overlap population because treatment recommendations are often conflicting in guideline recommendations for COPD patients versus pure bronchiectasis patients. Conversely, macrolide antibiotics have appeared to be effective in patients with bronchiectasis and frequent exacerbations however there continue to be some concerns about the possible use of macrolides and their ability to induce significant cardiovascular adverse effects, ${ }^{17}$ particularly in COPD patients who are already at risk of higher cardiovascular comorbidity. Furthermore, there is the concern about the development of multiantibiotic resistance particularly given the reported prevalence of nontuberculous mycobacterial (NTM) infections in COPD patients. ${ }^{18}$ In this Journal club we review some recent papers that have addressed some of these issues. Suffice it to say that additional prospective trial research is much required.

Note: Abstracts are presented in their original, published format and have not been edited to match JCOPDF style.

\section{Abstract 1 Bronchiectasis in Patients Hospitalized With Acute Exacerbations of COPD in Spain: Influence on Mortality, Hospital Stay, and Hospital Costs (2006-2014) According to Gender}

Sánchez-Muñoz G, Lopez-de-Andrés A, Hernández-Barrera V, et al. PLoS One. 2019;14(1). e0211222.

doi: https://doi.org/10.1371/journal.pone.0211222

\section{Purpose}

The objectives of this study were to analyze the characteristics of male and female patients hospitalized with acute exacerbation of chronic obstructive pulmonary disease (AE-COPD) during 2006-2014 according to the presence or absence of bronchiectasis and to study the factors associated with in-hospital mortality (IHM) in patients hospitalized 
with AE-COPD and concomitant bronchiectasis.

\section{Methods}

We used the Spanish National Hospital Database to analyze patients admitted with AE-COPD as their primary diagnosis. Patients included in the study were stratified according to the presence or absence of bronchiectasis as their secondary diagnosis.

\section{Results}

We identified 386,646 admissions for AE-COPD, of which 19,679 (5.09\%) involved patients with concomitant bronchiectasis. When patients with and without bronchiectasis were compared, we observed that the incidence of infection by Pseudomonas aeruginosa was substantially higher in the former, as were the mean stay, cost, and percentage of readmissions, although IHM and comorbidity were lower. The course of patients with AE-COPD and bronchiectasis was characterized by a gradual increase in prevalence and mean age among men and no differences in prevalence or lower mean age in women. Mortality was $4.24 \%$ and $5.02 \%$ in patients with and without bronchiectasis, respectively, although significance was lost after a multivariate adjustment (OR 0.94; 95\% CI, 0.88-1.01). The factors associated with IHM were older age, higher comorbidity, isolation of $P$. aeruginosa, mechanical ventilation and readmission.

\section{Conclusions}

The prevalence of admission with AE-COPD and bronchiectasis increased in men but not in women during the study period. In patients hospitalized with AE-COPD, we did not find differences in mortality when comparing the presence and absence of bronchiectasis. The analysis of temporal trends revealed a significant reduction in mortality from 2006 to 2014 in male patients with COPD and concomitant bronchiectasis, but not among women. It is important to consider the factors associated with IHM such as age, comorbidity, isolation of $P$. aeruginosa, mechanical ventilation and readmission to better identify those patients who are at greater risk of dying during hospitalization.

\section{Comments}

In this study, data collection is retrospective from the Spanish National Hospital Database. The prevalence rates reported in this study appear to reflect admission events and not the actual prevalence of bronchiectasis in COPD patients. As such, certain individuals may be represented numerous times in the data set. Furthermore, the diagnoses are based on diagnostic coding and there is no assurance that diagnoses for COPD and/ or bronchiectasis were based on spirometry or computed tomography (CT) scans respectively. Cost calculations were estimates using diagnosisrelated groups for the disease. Considering that many individuals may have had several admissions over the 8-year period of this study, the reduction in mortality over the length of the study may reflect a survivor bias. Interestingly, the investigators also noted a decrease in the prevalence of pneumococcal infections and they proposed this may reflect increases in pneumococcal vaccination but this was not confirmed. The group also reported that cigarette smoking seemed to have a protective effect with regard to reduced mortality but given the retrospective nature of the study they could only speculate that this may reflect more severe patients quitting smoking over time. Despite these limitations, this study, with its large database, still provides valuable insights into the trends for patients with COPD and bronchiectasis and the factors that may be associated with increased in-hospital mortality. Prospective trials that collect appropriate data to confirm diagnoses and follow outcomes of individuals are needed to better define risk factors and prognosis.

\section{Abstract 2 \\ Co-existence of COPD and Bronchiectasis: A Risk Factor for a High Ratio of Main Pulmonary Artery to Aorta Diameter (PA: A) from Computed Tomography in COPD Patients}

Dou S, Zheng C, Ji S, et al. Int. J Chron Obstruct Pulmon Dis. 2018;13:675-681.

doi: https://doi.org/10.2147/COPD.S156126

\section{BACKGROUND:}

Pulmonary vascular disease, especially pulmonary 
hypertension, is an important complication of COPD. Bronchiectasis is considered not only a comorbidity of COPD, but also a risk factor for vascular diseases. The main pulmonary artery to aorta diameter ratio (PA:A ratio) has been found to be a reliable indicator of pulmonary vascular disease. It is hypothesized that the co-existence of COPD and bronchiectasis may be associated with relative pulmonary artery enlargement (PA:A ratio $>1$ ).

\section{METHODS:}

This retrospective study enrolled COPD patients from 2012 through 2016. Demographic and clinical data were collected. Bhalla score was used to determine the severity of bronchiectasis. Patient characteristics were analyzed in two ways: the high $(\mathrm{PA}: \mathrm{A}>1)$ and low (PA:A $\leq 1)$ ratio groups; and COPD with and without bronchiectasis groups. Logistic regression analysis was used to assess risk factors for high PA:A ratios.

\section{RESULTS:}

In this study, 480 COPD patients were included, of whom 168 had radiographic bronchiectasis. Patients with pulmonary artery enlargement presented with poorer nutrition (albumin, 35.6 \pm 5.1 vs $38.3 \pm 4.9$, $P<0.001)$, lower oxygen partial pressure $(74.4 \pm 34.5$ vs $81.3 \pm 25.4, \quad P<0.001)$, more severe airflow obstruction (FEV1.0, $0.9 \pm 0.5$ vs $1.1 \pm 0.6, P=0.004$ ), and a higher frequency of bronchiectasis ( $60 \%$ vs $28.8 \%, P<0.001)$ than patients in the low ratio group. Patients with both COPD and bronchiectasis had higher levels of systemic inflammation (erythrocyte sedimentation rate, $P<0.001$ and fibrinogen, $P=0.006$ ) and $\mathrm{PA}$ : $\mathrm{A}$ ratios $(P<0.001)$. $\mathrm{A}$ higher $\mathrm{PA}$ : $\mathrm{A}$ ratio was significantly closely correlated with a higher Bhalla score $(\mathrm{r}=0.412, P<0.001)$. Patients with both COPD and bronchiectasis with high ratios presented higher levels of NT-proBNP $(P<0.001)$ and systolic pulmonary artery pressure $(P<0.001)$. Multiple logistic analyses have indicated that bronchiectasis is an independent risk factor for high PA:A ratios in COPD patients $(\mathrm{OR}=3.707$; 95\% CI $=1.888-7.278$; $P<0.001)$.

\section{CONCLUSION:}

Bronchiectasis in COPD has been demonstrated to be independently associated with relative pulmonary artery enlargement.

\section{Comments}

Cardiovascular comorbidity in COPD is well recognized and as the authors point out, pulmonary hypertension in COPD patients is associated with poorer prognosis, increased risk of exacerbations and death. Although the authors acknowledge that the actual mechanism(s) remains unknown, they note that vascular diseases in general, appear to be more common in patients with bronchiectasis and these investigators hypothesize that the airway injury and consequent repair process of bronchiectasis also leads to vascular damage, repair and remodeling. They suggest that hypoxia and vascular stiffness may be other mechanisms associated with vascular disease in COPD patients with or without bronchiectasis. This is a retrospective study involving review of CT scans and clinical and radiographic data. The Naidich criteria were used to diagnose bronchiectasis on CT scans and individuals with radiographic evidence suggesting other causes for pulmonary hypertension were excluded. Echocardiograms were apparently done but there is no report of any attempt to assess the correlation of the pulmonary artery to aorta (PA/A) ratio and signs of elevated right ventricle systolic pressures or right ventricular changes. Individuals did not routinely have right heart catheterization (RHC) data to evaluate the correlation between the $\mathrm{PA} / \mathrm{A}$ ratio and RHC measurements. Approximately $25 \%$ had ischemic heart disease and approximately $30 \%$ had systemic hypertension. It is unfortunate that "pulmonary function testing" was actually only spirometry without lung volumes or diffusing capacity and the investigators did not evaluate the extent of emphysema on CT scans of the chest. Considering the presence of vascular hypertension in at least $30 \%$ of the individuals, this could render the bronchial wall internal diameter/adjacent blood vessel diameter ratio problematic to use as a criterion to identify bronchiectasis. They did use the other criteria such as lack of bronchial tapering, visualization of peripheral bronchi within $1 \mathrm{~cm}$ of the costal pleura or in contact with the mediastinal pleura and the other criteria mentioned above in the introduction section.

The PA/A ratio is a measurement to detect possible pulmonary hypertension in patients with evidence of COPD and bronchiectasis. Such patients show higher values for several systemic inflammatory indices and appear to have a poorer prognosis. The 
study demonstrates the complex inter-relationships between COPD, bronchiectasis and pulmonary hypertension and points to the need to investigate whether COPD patients with bronchiectasis may have concomitant pulmonary hypertension that may confer a worse prognosis. Prospective studies that document other comorbidities, exacerbation frequency, and microbial load in true COPD patients with a relevant exposure history are required to better understand the relationships and possible mechanisms.

\section{Abstract 3 Association Between Inhaled Corticosteroid Use and Pulmonary Nontuberculous Mycobacterial Infection}

Liu VX, Winthrop KL, Lu Y, Sharif H, Nasiri HU, Ruoss SJ. Ann Am Thorac Soc. 2018;15(10):1169-1176.

doi: https://doi.org/10.1513/AnnalsATS.201804-245OC

\section{RATIONALE:}

Nontuberculous mycobacterial (NTM) pulmonary disease prevalence is increasing.

\section{OBJECTIVES:}

To determine the association between the use of inhaled corticosteroids and the likelihood of NTM pulmonary infection among individuals with treated airway disease.

\section{METHODS:}

We conducted a case-control study of subjects with airway disease with and without NTM pulmonary infection (based on mycobacterial respiratory cultures) between 2000 and 2010 in northern California. We quantified the use of inhaled corticosteroids, other airway disease medications, and healthcare use within 6 months of NTM pulmonary infection identification. We used 1:10 case-control matching and conditional logistic regression to evaluate the association between the duration and cumulative dosage of inhaled corticosteroid use and NTM pulmonary infection.

\section{RESULTS:}

We identified 248 cases with NTM pulmonary infection with an estimated rate of 16.4 cases per
10,000 subjects treated for airway disease. The median interval between treated airway disease cohort entry (defined as date of patient filling the third airway disease treatment prescription) and NTM case identification was 1,217 days. Compared with control subjects, subjects with NTM pulmonary infection were more likely to use airway disease medications including systemic steroids; they were also more likely to use health care. Any inhaled corticosteroids use between 120 days and 2 years before cohort entry was associated with substantially increased odds of NTM infection. For example, the adjusted odds ratio for NTM infection among inhaled corticosteroid users in a 2-year interval was 2.51 (95\% confidence interval, 1.40-4.49; $\mathrm{P}<0.01$ ). Increasing cumulative inhaled corticosteroid dose was also associated with greater odds of NTM infection.

\section{CONCLUSIONS:}

Inhaled corticosteroid use, and particularly high-dose inhaled corticosteroid use, was associated with an increased risk of NTM pulmonary infection.

\section{Comments}

The authors point out that the prevalence of pulmonary NTM infections in the 1980s was reported to be as high as 1.8 cases per 100,000 persons, in the 1990 s around 3-4 cases per 100,000 and recent studies report the rate as high as 40/100,000. This coincides with the introduction of inhaled corticosteroids (ICSs) in the 1980s in North America and late 1970s in Europe. While this is a retrospective study it demonstrates quite a compelling association between ICS use and evidence of a dose response relationship with increased cumulative dose of ICS and evidence of positive cultures for NTM. In this study, each month of high dose ICS use was independently associated with greatly increased odds of developing NTM pulmonary infections. This cohort contained a mixture of patients with asthma (59.7\%), COPD (29.4\%) and bronchiectasis (10.9\%). Further, $30.6 \%$ had more than one diagnosis, most commonly COPD and asthma. Cystic fibrosis patients and patients with a prior history of tuberculosis were excluded. The higher proportion of asthma patients than COPD and bronchiectasis patients may further support the association of ICS use and NTM positive cultures as a higher proportion of asthma patients are on ICS or ICS/long-acting beta2-agonists (LABA) 
combinations compared to COPD and bronchiectasis patients. The NTM case cohort had $92 \%$ on ICS or ICS/LABA and $36.3 \%$ on systemic steroids whereas the case matched control group had $51 \%$ on ICS or ICS/LABA and $14.2 \%$ on systemic steroids. Case identification was based on 2 positive sputum cultures within a year and/or 1 positive bronchoscopy culture. Interestingly they based NTM identification in each patient only on basis of the first culture and did not document if the cultured organism changed in the 2 sputum cultures. The latter scenario may point more to an example of dysbiosis rather than true pulmonary disease. NTM organisms most frequently identified were M. avium-intracellulare (67\%), M. Gordonae (16.6\%), M. Abscessus (5.3\%), M. fortuitum (4.6\%), M. Chelonae (3.5\%) and M. Kansasi (3.5\%). Patients that had M. Gordonae and/or had positive cultures before starting on corticosteroids were excluded from analysis. The authors examined comorbidities including gastroesophageal reflux disease, diabetes, interstitial lung disease and rheumatoid arthritis and there was slightly higher use of anti-TNF agents $(0.8 \%$ versus $0.1 \%$ ) and other immunosuppresants (4.4\% versus $2.2 \%$ ) for the NTM case group versus controls respectively. The contribution of these agents to the NTM cases was relatively small compared to the use of inhaled corticosteroids and systemic steroids.

The authors do point out that they only use the American Thoracic Society/ Infectious Diseases Society of America ${ }^{19}$ consensus microbiological criteria to identify patients with NTM infection. They did not assess whether individuals met the consensus definition of NTM pulmonary disease. They do point out however, that prior published work established that the microbiological disease criteria were valid as a surveillance tool with a roughly $85 \%$ concordance of microbiological disease definition with clinical disease definition. ${ }^{20}$ They also note that prognosis based on microbiological and NTM infection criteria is also highly concordant with the prognosis of those meeting the consensus NTM clinical disease definition. ${ }^{21}$ They also indicated that they did not look to see if there was a difference between different inhaled corticosteroids used by the patient population and made dose equivalent adjustments using broadly accepted standard ICS equivalence adjustments to beclomethasone. They also offered that they doubted that there was a difference in specific ICSs used by patients that was likely to account for the results reported. It is worth noting however, that there have been previous studies suggesting that there may be differences between corticosteroids in terms of the expression of certain immune defense genes. ${ }^{22}$ Finally, with the use of a case-control approach one cannot establish a causal link between ICS use and NTM pulmonary infections.

\section{Bottom Line}

The COPD/bronchiectasis overlap defines a group of COPD patients who generally appear to have more severe disease and poorer prognosis. These patients tend to demonstrate greater symptoms, lower lung function, frequent exacerbations and are at greater risk of developing pulmonary hypertension. The underlying pathobiology remains unclear as to whether one causes the other and what the direction of causation may be. Bronchiectasis has many potential causes including cystic fibrosis, alpha-1 antitrypsin deficiency, aspiration, and these should be ruled out or treated if present. Identifying groups at high risk and early detection are key. There remain many questions to answer concerning treatment and prevention including the role of inhaled steroids, macrolides and inhaled antibiotics, PDE4 inhibitors, mucolytics, etc. Airway clearance has been proven to be an important part of treatment. Rather than considering such overlaps as syndromes it is perhaps best to consider them and manage them as treatable traits. Prospective studies are needed to best address these issues. 


\section{References}

1. Polverino E, Dimakou K, Hurst J, et al. The overlap between bronchiectasis and chronic airway diseases: state of the art and future directions. Eur Respir J. 2018;52(3).

doi: https://doi.org/10.1183/13993003.00328-2018

2. Sethi S, Murphy TF. Infection in the pathogenesis and course of chronic obstructive pulmonary disease. $N$ Engl J Med. 2008;359(22):2355-2365.

doi: https://doi.org/10.1056/NEJMra0800353

3. Wang Z, Singh R, Miller BE, et al. Sputum microbiome temporal variability and dysbiosis in chronic obstructive pulmonary disease exacerbations: an analysis of the COPDMAP study. Thorax. 2018;73(4):331-338.

doi: https://doi.org/10.1136/thoraxjnl-2017-210741

4. Naidich DP, McCauley D, Khouri N, Stitik F, Siegelman S. Computed tomography of bronchiectasis. J Comput Assist Tomogr.1982;6(3):437-444.

doi: https://doi.org/10.1097/00004728-198206000-00001

5. Kocurek EG, Jagana R. Noncystic fibrosis bronchiectasis management: opportunities and challenges. Curr Opin Pulm Med. 2019;25(2):192-200.

doi: https://doi.org/10.1097/MCP.0000000000000562

6. Cole PJ. Inflammation: a two-edged sword-the model of bronchiectasis. Eur J Respir Dis.1986;147(Suppl):6-15.

7. Martinez-Garcia MA, Miravitlles M. Bronchiectasis in COPD patients: more than a comorbidity? Int J Chron Obstruct Pulmon Dis. 2017;12:1401-1411.

doi: https://doi.org/10.2147/COPD.S132961

8. Lonni S, Chalmers JD, Goeminne PC, et al. Etiology of noncystic fibrosis bronchiectasis in adults and its correlation to disease severity. Ann Am Thorac Soc. 2015; 12(12):1764-1770. doi: https://doi.org/10.1513/AnnalsATS.201507-472OC

9. Mao B, Yang J-W, Lu H-W, Xu J-W. Asthma and bronchiectasis exacerbation. Eur Respir J. 2016;47(6):1680-1686. doi: https://doi.org/10.1183/13993003.01862-2015

10. Guilemany JM, Angrill J, Alobid I, et al. United airways again: high prevalence of rhinosinusitis and nasal polyps in bronchiectasis. Allergy. 2009;64(5)790-797.

doi: https://doi.org/10.1111/j.1398-9995.2008.01892.x

11. Lee AS, Ryu JH. Aspiration pneumonia and related. Mayo Clin Proc. 2018;93(6):752-762.

doi: https://doi.org/10.1016/j.mayocp.2018.03.011

12. Brode SK, Campitelli MA, Kwong JC, et al. The risk of mycobacterial infections associated with inhaled corticosteroid use. Eur Respir J. 2017;50(3):1700037.

doi: https://doi.org/10.1183/13993003.00037-2017
13. Haldar K, Bafadehl M, Lau K, et al. Microbiome balance in sputum determined by PCR stratifies COPD exacerbations and shows potential for selective use of antibiotics. PLoS One. 2017;12(8): e0182833.

doi: https://doi.org/10.1371/journal.pone.0182833

14. Pittman JE, Wylie KM, Akers K, et al. Association of antibiotics, airway microbiome, and inflammation in infants with cystic fibrosis. Ann Am Thorac Soc. 2017;14(10):1548-1555. doi: https://doi.org/10.1513/AnnalsATS.201702-121OC

15. Tufvesson E, Markstad H, Bozovic G, Ekberg M, Bjermer L. Inflammation and chronic colonization of Haemophilus influenzae in sputum in COPD patients related to the degree of emphysema and bronchiectasis in high-resolution computed tomography. Int J Chron Obstruct Pulmon Dis. 2017;12:32113219. doi: https://doi.org/10.2147/COPD.S 137578

16. Kapur N, Bell S, Kolbe J, Chang AB. Inhaled steroids for bronchiectasis. Cochrane Database Syst Rev. 2009;1:CD000996. doi: https://doi.org/10.1002/14651858.CD000996.pub2

17. Kang J, Kim Y-J, Shim TS, Jo K-W. Risk for cardiovascular disease in patients with nontuberculous mycobacteria treated with macrolide. J Thorac Dis. 2018;10(10):5784-5795. doi: https://doi.org/10.21037/jtd.2018.09.145

18. Mustafa MH, Khandekar S, Tunney MM, et al. Acquired resistance to macrolides in Pseudomonas aeruginosa from cystic fibrosis patients. Eur Respir J. 2017;49(5). doi: https://doi.org/10.1183/13993003.01847-2016

19. Griffith DE, Aksamit T, Brown-Elliott BA, et al. ATS Mycobacterial Diseases Subcommittee; American Thoracic Society; Infectious Diseases Society of America. An official ATS/IDSA statement: diagnosis, treatment, and prevention of nontuberculous mycobacterial diseases. Am J Respir Crit Care Med. 2007;175:367-416. [Published erratum appears in Am J Respir Crit Care Med. 2007;175:744-745.]

20. Brassard P, Suissa S, Kezouh A, Ernst P. Inhaled corticosteroids and risk of tuberculosis in patients with respiratory diseases. Am J Respir Crit Care Med. 2011;183(5): 675-678. doi: https://doi.org/10.1164/rccm.201007-10990C

21. Winthrop KL, McNelley E, Kendall B, et al. Pulmonary nontuberculous mycobacterial disease prevalence and clinical features: an emerging public health disease. Am J Respir Crit Care Med. 2010;182(7):977-982. doi: https://doi.org/10.1164/rccm.201003-0503OC

22. van den Berge M, Jonker MR, Miller-Larsson A, Postma DS, Heijink I. Effects of fluticasone propionate and budesonide on the expression of immune defense genes in bronchial epithelial cells. Pulm Pharmacol Ther. 2018;50: 47-56. doi: https://doi.org/10.1016/j.pupt.2018.04.002 